\title{
EchoGéo
}

36 | 2016

Stratégies de villes et "modèles urbains"

\section{La ville fonctionnelle et les modèles urbains qui lui ont succédé}

Exemples d'une pratique politique en train de se globaliser

Herman van der Wusten

\section{CpenEdition}

\section{Journals}

Electronic version

URL: https://journals.openedition.org/echogeo/14634

DOI: $10.4000 /$ echogeo.14634

ISSN: 1963-1197

Publisher

Pôle de recherche pour l'organisation et la diffusion de l'information géographique (CNRS UMR 8586)

Electronic reference

Herman van der Wusten, "La ville fonctionnelle et les modèles urbains qui lui ont succédé", EchoGéo

[Online], 36 | 2016, Online since 30 June 2016, connection on 10 August 2021. URL: http://

journals.openedition.org/echogeo/14634 ; DOI: https://doi.org/10.4000/echogeo.14634

This text was automatically generated on 10 August 2021.

EchoGéo est mis à disposition selon les termes de la licence Creative Commons Attribution - Pas

d'Utilisation Commerciale - Pas de Modification 4.0 International (CC BY-NC-ND) 


\title{
La ville fonctionnelle et les modèles urbains qui lui ont succédé
}

\author{
Exemples d'une pratique politique en train de se globaliser
}

\author{
Herman van der Wusten
}

Je remercie Virginie Mamadouh pour son assistance à la rédaction de ce texte en français

1 Il y a au moins deux genres de modèles urbains. L'un renvoie à des descriptions théoriques (autant que possible en termes quantitatifs) qui peuvent expliquer des aspects essentiels de la morphologie ou du fonctionnement des villes (Glaeser, 1992 ; Harding et Blokland, 2014) ; l'autre aux représentations des traits essentiels d'une ville idéale à réaliser. Cette contribution s'intéresse aux modèles urbains de la deuxième catégorie et en particulier à ceux qui sont apparus dans le discours public et se sont insérés dans les pratiques de la gestion urbaine au cours des dernières décennies (Peck, 2010), depuis le déclin du modèle urbain de Le Corbusier et du CIAM, point culminant de l'urbanisme moderniste. Il s'agit alors de répondre à la question de savoir comment faire évoluer la/notre ville?

2 Plusieurs modèles ont été proposés et discutés, puis ont servi de ligne conductrice pour les pratiques d'urbanisme. La littérature générale sur l'évolution de l'urbanisation et de l'urbanisme, et la documentation des organisations internationales dans ce domaine le démontrent. Les exemples qui suivent sont bien connus, mais il n'est pas certain que la liste soit exhaustive et il y a probablement des expériences ici ou là qui n'ont pas été recensées. Par conséquent, cet inventaire de cas est biaisé, puisqu'il présente les cas les plus connus qui ne sont pas nécessairement les plus réussis ; au cours de cet exercice de nature exploratoire, je m'intéresse tout d'abord à l'origine et à la diffusion de ces modèles.

3 La diffusion des innovations était un des sujets privilégiés de la géographie quantitative des années 1960. Il s'agissait alors de l'adoption de biens tangibles récemment inventés, par des individus après qu'ils aient été suffisamment informés de leur existence et de leur disponibilité. La production, la circulation et l'adoption de "modèles urbains " s'inscrit dans une logique apparemment similaire qui présente cependant des différences importantes. En effet, il ne s'agit pas, dans la plupart des cas, de biens 
tangibles et uniques, mais en général d'une série d'éléments liés, lesquels peuvent être des biens matériels mais aussi des institutions, des pratiques et des prescriptions. Ils ont tendance à se modifier au cours de leur circulation et de leur adoption, et sont finalisés au travers de programmes et de stratégies politiques.

4 Les succès et les échecs des modèles courants sont l'objet d'un dialogue régulier entre les villes et les institutions en charge de la pratique politique qui les concernent dans le contexte actuel d'une intensification des relations. Producteurs, initiateurs ou avocats du modèle (des individus, quelquefois un mouvement) l'adaptent à l'évolution des conditions. Ainsi un modèle est-il tout à la fois diffusé et transformé au cours du processus (McCann et Ward, 2011). Il y a un problème de continuité.

5 Cette contribution s'intéresse aux modèles urbains introduits depuis la première partie $\mathrm{du}$ XXe siècle pour réaliser des villes plus attrayantes. Les modèles plus récents sont décrits et comparés au modèle de la ville fonctionnelle du CIAM et de Le Corbusier. Les individus et institutions qui ont pris l'initiative de construire et introduire ces modèles sont identifiés et quelques informations sur la diffusion de ces modèles et leurs transformations au cours du processus sont indiquées.

\section{Les modèles urbains : une typologie}

6 Les modèles urbains mis en œuvre dans beaucoup de villes autour du monde suggèrent des approches assez concrètes et servent comme fils conducteurs aux pratiques urbaines. Ils sont l'occasion de slogans mobilisateurs - souvent hégémoniques, parfois de l'ordre des proclamations polémiques dans des luttes verbales - qui sont au cœur des pratiques politiques urbaines.

Illustration 1 - Portée et approche des modèles urbains de CIAM IV et de modèles proposés par la suite

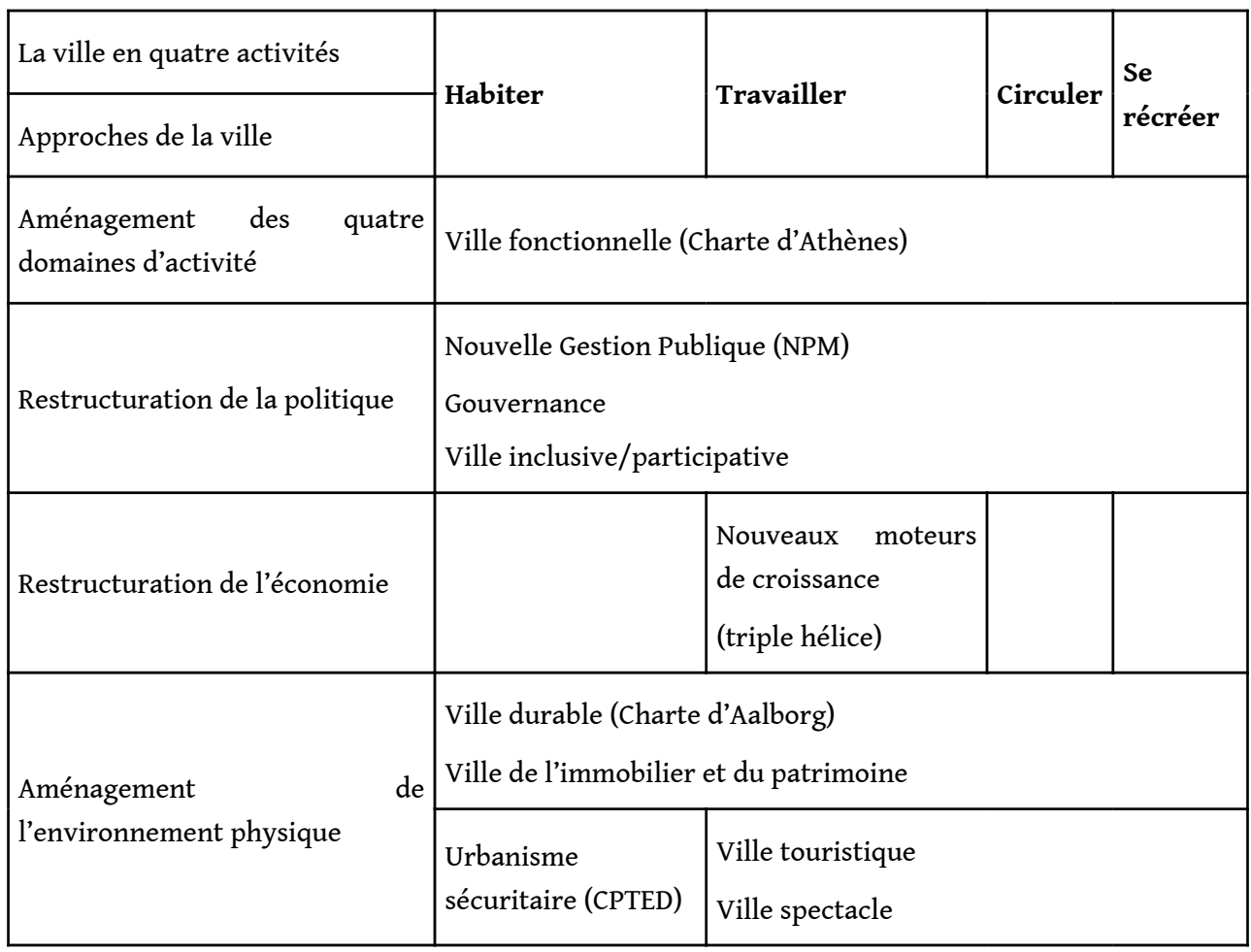


\begin{tabular}{ll|l}
$\begin{array}{l}\text { Développement de la ville } \\
\text { comme organisme social }\end{array}$ & Ville intelligente \\
Ville créative
\end{tabular}

7 Cette typologie fait l'inventaire des modèles urbains à analyser. Elle est fondée sur deux dimensions que sont leur portée et leur approche du phénomène urbain. La portée de chaque modèle est analysée à travers les quatre domaines d'activités que Le Corbusier et le CIAM utilisaient pour construire leur modèle de la ville fonctionnelle : habiter, travailler, circuler et se récréer.

8 Par ailleurs, la typologie caractérise les approches de la ville qui s'expriment dans les modèles urbains proposés dans l'exemple moderniste et dans les suivants. Tandis que les producteurs de ce modèle initial percevaient la ville comme la scène où se tenaient de multiples activités déclinées en 4 genres, les trois catégories de modèles suivants se concentrent sur un seul aspect de la réalité urbaine qu'ils considèrent comme de loin le plus important : celui de la politique, l'économie et l'environnement physique. Enfin, il y a une catégorie de modèles qui, comme celui de Le Corbusier, s'intéressent à la ville dans son intégralité. Mais l'objectif n'est pas alors un aménagement physique optimal pour les différents domaines d'activités. Il s'agit d'obtenir les meilleures conditions pour atteindre un stade de développement supérieur de la société urbaine locale - soit un ensemble d'individus qui entretiennent entre eux des liens mutuels développés et aux conséquences vertueuses.

9 Les modèles de cette typologie ont une portée différente pour les quatre domaines d'activités dans la ville identifiés par Le Corbusier. Certains touchent à tous ces domaines d'activités. D'autres, plus sélectifs, considèrent les domaines retenus comme emblématiques de la ville toute entière.

\section{Aménager les quatre domaines d'activités : la pratique CIAM}

10 Le modèle urbain de Le Corbusier et du CIAM a pour objectif d'adapter la ville aux exigences de la modernité, à la suite de l'industrialisation et des avancées technologiques des premières décennies $\mathrm{du} \mathrm{XX}^{\mathrm{e}}$ siècle. Le modèle a été formulé en opposition totale aux points de vue habituellement adoptés par l'urbanisme à l'époque. La ville fonctionnelle est l'objectif de ce modèle. Les quatre grands domaines d'activités humaines (habiter, travailler, circuler et se récréer) s'expriment dans des zones distinctes à l'intérieur de la ville; celles-ci sont aménagées d'une façon efficace et efficiente en utilisant les technologies les plus modernes disponibles pour la construction. Le premier Congrès International de l'Architecture Moderne (CIAM), vecteur de cette pensée, s'est tenu en 1928 ; le dernier en 1959. Le quatrième en 1933 s'est terminé à Athènes, après une excursion maritime en Méditerranée. Il a été l'occasion de nombreux débats au cours desquels la grille d'une Charte d'Athènes fut mise au point. Cette grille prit sa forme définitive sous la plume de Le Corbusier en 1941 (Le Corbusier 1942).

11 La Charte d'Athènes a été rédigée après l'analyse de 33 plans de villes qui étaient présentés à Athènes par les participants au congrès. Ils nous donnent une première impression de la diffusion des idées de la Charte à ses débuts. À partir de ces 33 plans 
(Corbusier, 1942, p. 95), Le Corbusier produisait une liste de 32 toponymes, dont 27 de toutes les parties de l'Europe, deux de villes nouvelles dans des colonies (Vietnam et Indonésie) et trois aux États-Unis. Les pays les mieux représentés sur cette liste sont l'Italie (cinq plans dont un d'une ville nouvelle récemment « inaugurée » par Mussolini) et l'Allemagne (quatre plans dont celui de Dessau, résidence du Bauhaus après son départ de Weimar, fermée en 1932 à la demande des nazis).

Le modèle proposé dans la Charte a joué un rôle considérable au moins jusqu'aux années 1960. Alors que Le Corbusier en rédigeait la version définitive, il préparait un plan similaire pour le développement d'Alger. Plus tôt, en 1934, un Plan de l'Extension Générale d'Amsterdam avait été préparé méticuleusement, achevé par un architecte du CIAM et un grand nombre de collaborateurs techniques de différentes disciplines (Van Rossem, 1993). Il fut mis en œuvre après-guerre. La ville fonctionnelle fut le modèle urbain dominant pendant la période de reconstruction massive en Europe de l'Est et en Europe de l'Ouest. À Amsterdam, après la réalisation du Plan de 1934, la phase suivante du développement urbain prévoyait une grande restructuration de la ville existante selon les idées du CIAM. Cette restructuration suscita une vive résistance vers la fin des années 1960 et fut finalement abandonnée. De nouveaux modèles urbains apparurent alors.

\section{La restructuration de la politique}

Par la suite, la vie urbaine est abordée par le biais de la politique. Cette dimension manquait évidemment dans la Charte, dont l'approche reste technocratique: l'aménagement y était placé sous la responsabilité de l'architecte et fondé sur un savoir technique. Son plan est la base de sa propre réalisation. Les gouvernements ne sont que des assistants utiles pour régler le zoning des fonctions inscrites dans le plan. En conclusion: "L'architecture préside aux destinées de la cité... L'architecture est à la clef de tout » (Le Corbusier, 1942, par. 85, p. 107-108; par 92, p. 114).

La politique se traduit dans les modèles urbains de l'ère post-corbuséenne selon des modalités différentes qui conduisent à trois versions de modèles. Chacune de ces versions se décline pour la ville mais aussi pour les relations avec d'autres entités sociales, en particulier celles avec l'État. Dans ces modèles, la ville est considérée comme une entité de nature politique (pour l'État, cela va de soi). Le rôle politique de la ville était minimisé, en particulier au moment de l'État-providence. La ville est maintenant de nouveau considérée comme un acteur politique, mais elle est en même temps étroitement liée à d'autres villes et à des acteurs actifs à d'autres échelles que celle de la ville. La ville contemporaine est donc fortement imbriquée dans des réseaux multiscalaires.

Les trois versions de l'approche politique dans les modèles urbains renvoient (i) à l'application de la "Nouvelle Gestion Publique" (New Public Management) dans la politique et l'administration existante de la ville; (ii) à l'application des principes de "Bonne Gouvernance" dans les pratiques de collaboration entre tous les parties intéressées dans la gestion de la ville à l'intérieur et à l'extérieur de l'administration urbaine/locale ; (iii) et à l'incorporation de tous les habitants d'une "Ville inclusive " dans la sphère publique, où les décisions collectives sont prises pour s'assurer que toutes les préférences et tous les intérêts sont pris en compte et que les services publics sont accessibles à tous. 
16 La Nouvelle Gestion Publique (NPM) souligne la capacité des dirigeants urbains à traduire les préférences de la population urbaine en "produits " appréciés. Les entrepreneurs et leurs clients sur le marché servent d'exemples. Les prescriptions de la NPM recommandent de renforcer l'autorité des managers et des techniciens de la gestion aux dépens de celle des politiciens. La formule a fait fortune pendant les années 1980 et 1990. Elle a démarré en Angleterre, et a été appliquée à tous les niveaux dans tous les domaines de la gestion publique et accompagnée de la privatisation des services publics. Appliquées partout dans le monde selon des versions différentes, les idées de la NPM ont été exportées depuis l'Angleterre vers le reste de l'OCDE puis audelà (Eymeri-Douzans et Pierre, 2011).

La notion de gouvernance, elle, a été introduite au début des années 1990 par des organisations internationales (l'ONU, la Banque mondiale, le FMI) pour faire de la qualité de l'action gouvernementale de leurs États membres un sujet de discussion (World Bank, 1991, Huynh-Quan-Suu s.a.). La bonne gouvernance a finalement été définie en termes de sélection des décideurs, de la capacité à prendre des décisions régulatrices contraignantes et de la légitimité des arrangements gouvernementaux (Independent Evaluation Group World Bank, 2011). Au cours de l'élaboration du concept la notion de la contribution indispensable d'acteurs positionnés en dehors du gouvernement au sens strict a été renforcée. Cette gouvernance prend la forme de séries de réseaux d'acteurs autonomes et interdépendants, orientés sur un problème qui demande une intervention de nature régulatrice. Ces réseaux ont un caractère horizontal et ils sont composés d'unités gouvernementales traditionnelles et d'autres parties prenantes (intérêts organisés, représentants reconnus de la société civile). Des accords doivent être obtenus au moyen de négociations, ainsi que de l'augmentation de la confiance mutuelle, de l'émergence de normes acceptées par tous, etc. Au cas où ces interactions resteraient sans résultat, il est alors fait appel à la méta-gouvernance. Il s'agit, en fait, de l'intervention du gouvernement dans le processus (Torfing, 2012).

Dans une troisième version, la politique doit fonctionner dans la «ville inclusive » comme pratique d'une vie urbaine qui inclut la totalité de la population urbaine. Elle est conçue comme le rassemblement de vrais citoyens qui tous ont une voix pour exprimer leurs intérêts en même temps que la garantie d'accès à un niveau de vie qui permette la participation à la vie urbaine. Pour sa réalisation, la participation active de la population est requise.

La notion de ville inclusive s'est propagée notamment à partir d'expériences menées dans des villes brésiliennes après l'introduction d'une nouvelle constitution en 1988 . Un exemple célèbre est celui du budget participatif à Porto Alegre qui a fonctionné avec quelque succès pendant la première décennie du XXI siècle (Langelier, 2011). Cette ville a aussi accueilli la première édition du Forum social mondial en 2001 et plusieurs éditions ultérieures de cet événement. Celui-ci met la démocratie directe et des services publics accessibles à tous au centre de ses préoccupations. Le Forum a été créé comme contrepoids au Forum économique mondial qui se tient tous les ans à Davos.

La notion de ville inclusive a été popularisée pendant la même période par le programme Habitat de l'ONU. Cette institution qui était, à l'origine en 1976, principalement orientée vers la fonction résidentielle de la ville s'est, au cours du temps, ouverte sur les autres fonctions urbaines et a finalement adopté une perspective plus ou moins politique. Dans un rapport important publié en 2001, le programme considère la planification urbaine et la prise de décisions participatives comme un trait 
essentiel de la ville inclusive. Une telle forme de gouvernance diminuerait l'inégalité et les tensions sociales. Elle peut incorporer la connaissance, la productivité et le capital social et physique des plus démunis et des plus désavantagés dans le processus de développement. Elle peut augmenter l'appropriation locale des processus et des programmes de développement (UN-Habitat, 2001).

21 Pourtant, au fil du temps, UN-Habitat n'a pas retenu le modèle de la ville inclusive comme la meilleure approche pour guider la ville dans son évolution, bien que l'organisation ait entretemps maintes fois réitéré l'importance de cette notion. Ces dernières années, UN-Habitat identifie une liste de sujets principaux qui touchent à une série de questions très diverses portant sur la sphère urbaine, sans distinction et sans modèle urbain préférentiel pour affirmer son identité. Elle sélectionne certains sujets sur cette liste à traiter en priorité durant plusieurs années (http://unhabitat.org/ about-us/goals-and-strategies-of-un-habitat/).

En Amérique Latine et dans d'autres pays en voie de développement, la Banque mondiale a très activement encouragé les pratiques de bonne gouvernance qui sont souvent un mélange de prescriptions dérivées des modèles NPM et de la Bonne Gouvernance et fondées sur des réseaux politiques. En fait, la Banque mondiale intervient dans ces réseaux, aussi bien que les subdivisions d'administrations locales et régionales, dans des projets pour améliorer le fonctionnement de services publics divers (Frey, 2008). C'est un exemple de réseau politique multiscalaire. La gouvernance récente des villes brésiliennes démarre souvent avec une version spécifique des modèles politiques que nous venons de présenter. Les pratiques politiques qui en ressortent éventuellement ont tendance à les mélanger (Frey et Duarte, 2006, par. 25).

Le rôle politique de la ville est donc (encore une fois) reconnu et l'approche politique fonctionne selon différentes versions mélangées dans un modèle urbain. Les versions de la NPM et de la bonne gouvernance s'enchaînent dans les pays anglo-saxons et les organisations mondiales, et se propagent ensuite vers les pays en voie de développement. L'OCDE joue un rôle dominant dans la diffusion vers les autres pays développés. Ces modèles sont de plus en plus mélangés (Klijn, 2012). En même temps, autour de l'année 2000, émerge un mouvement de démocratisation qui se traduit dans une troisième version de modèle politique. Ici et là, celle-ci est aussi plus ou moins mélangée aux autres modèles.

\section{La restructuration de l'économie}

La typologie (illustration 1) présente ensuite une série de modèles urbains orientés vers des changements dans les structures des économies développées. Dans la terminologie corbuséenne, il s'agit exclusivement d'activités à l'intérieur du domaine "travailler ». Une économie industrielle a été remplacée par une économie de services. Les chaînes de production se sont globalisées et les entreprises sont devenues multinationales.

Des modèles urbains se sont développés autour de l'attrait de certaines de ces activités. Ils ont joué un rôle dominant dans la littérature sur les villes mondiales initiée par John Friedmann (1986) et élaborée notamment par Sassen (1991) et Taylor (2004). Ils renvoient notamment aux concentrations de services avancés destinés aux entreprises (services financiers et juridiques, relations publiques) et des centres logistiques, ainsi 
qu'aux concentrations des sièges principaux de firmes multinationales et des centres de recherche.

Enfin, pour mener une politique urbaine d'innovation réussie, des alliances entre le gouvernement urbain, les universités locales et le secteur privé ont été proposées : la triple hélice (Etzkowitz, 2014). C'est encore une fois une gouvernance basée sur les réseaux qui est promue ici.

\section{L'aménagement de l'environnement physique}

La qualité et la profitabilité de l'environnement urbain ont été aussi à la base d'une série de modèles urbains divers. L'environnement inclut ici le fondement matériel de la vie urbaine (l'atmosphère, le sol, la nature, les constructions et les infrastructures). La "ville durable » est une notion introduite à la fin de la conférence de Rio de Janeiro organisée en 1992 par l'ONU. Pendant cette conférence, essentiellement entre États, mais avec la participation vivante d'ONG et de collectivités territoriales, il fut convenu que l'Agenda 21 avait besoin d'Agendas 21 locaux complémentaires pour progresser vers une situation planétaire durable. Les années suivantes verront une série d'initiatives au niveau mondial qui conduiront à une série d'initiatives pour produire des Agendas 21 locaux. En 1996, Maurice Strong, secrétaire-général de la conférence de Rio, par ailleurs entrepreneur à l'échelle mondiale et conseiller des secrétairesgénéraux de l'ONU pendant des décennies, mentionnait 1300 administrations locales qui, à cette date, avaient préparé leur propre Agenda 21 (ICLEI, 1996).

La notion de durabilité était dans ce contexte étendue à trois grands domaines: l'écologie, le développement économique et le développement communautaire, avec des interrelations supposées. La notion de ville durable fut élaborée plus tard, en particulier en Europe, et introduite dans la Charte d'Aalborg (1994). Elle soulignait l'axe écologique. La Charte fut établie comme antinomique à la Charte d'Athènes: elle promouvait la densité élevée et la mixité spatiale des fonctions, au lieu de leur séparation, elle encourageait les partenariats populaires dans la gestion au lieu de la technocratie. Au niveau des États, la France introduisit en 2008 une politique nommée «Ville durable » dans une logique similaire. L'Europe n'est pas le seul endroit où de telles initiatives étaient prises. Vancouver fait des efforts pour diminuer l'émission locale de dioxyde de carbone depuis 1990 et travaille avec un «Greenest City Action Plan » intégral depuis 2009.

Une approche comparable, bien que très différemment inspirée, perçoit la ville au travers de son parc immobilier et de son patrimoine. L'embellissement du patrimoine immobilier (chacun selon son goût) doit bénéficier à ses initiateurs et à la ville toute entière. Ainsi, par exemple, le réaménagement de zones portuaires délaissées avec la construction d'équipements et de logement afin d'en transformer les rives en feront des zones attractives et recherchées par les classes aisées (Boubacha, 1997, p. 15-30).

Trois autres modèles urbains abordent aussi la ville par son environnement physique, mais ils sont tous de portée plus limitée. La ville touristique et la ville spectacle affectent surtout les activités concernant le travail, les loisirs et la circulation. Évidemment, les modèles de villes touristiques et de villes spectacles ont tendance à se mélanger. 
31 Les villes touristiques dépendent presque toutes de leur environnement physique naturel ou de leur patrimoine. Pour les différents types de stations balnéaires, des modèles spéciaux ont été développés avec des promoteurs privés.

Les villes spectacles s'organisent autour des calendriers de spectacles annuels, que ce soient des festivals d'art, des foires pour des produits variés mais aussi Davos et Porto Alegre que nous avons déjà mentionnés ou un événement sportif (Le Mans). Certains directeurs de spectacles se sont installés durant une longue période dans la ville et ont marqué l'image de la ville en question. La famille Wagner et l'impact des Festspiele sur le profil de Bayreuth, où ils ont lieu chaque été en est un exemple extraordinaire.

Il y a aussi des villes spectacles temporaires, ayant obtenu le droit d'organiser un évènement unique comme les Jeux Olympiques, le championnat du monde pour un certain type de sport, organisé chaque fois dans une ville différente. Ces spectacles sont organisés selon des formats largement prescrits par les organisations et adaptés aux expériences antérieures. La procédure de sélection est de plus en plus sophistiquée et des services spécialisés de l'organisateur (par exemple le Comité Olympique International) s'occupent intensivement des préparations qui durent pendant des années. La ville et les services de l'organisation mondiale mis à part, le gouvernement national et les acteurs mondiaux, comme les chaînes de télévision, sont aussi des parties prenantes importantes. En conséquence, un réseau politique émerge, semblable à ceux qualifiés précédemment de bonne gouvernance; il est cette fois mis en place pour un projet de quelques années, qui peut donner des résultats très variables (pour Barcelone 1992, voir Moraga Spà et Botella, 1995). Le cas des Jeux Olympiques de Londres a été analysé de ce point de vue par Acuto (2013).

L'organisation de spectacles gigantesques rencontre de plus en plus souvent une opposition forte de la part des populations locales. Il devient difficile d'organiser les Jeux Olympiques - ceux d'été ou ceux d'hiver - dans l'hémisphère Nord en raison des protestations locales. Les habitants des villes spectacles candidates considèrent que leur vie urbaine sera trop perturbée par ces évènements, et ne se montrent guère sensibles aux perspectives présentées comme avantageuses par les organisateurs de l'évènement. Il s'agit donc d'un modèle urbain de plus en plus contesté.

troisième modèle, un urbanisme sécuritaire associé à l'acronyme CPTED est particulièrement engagé dans le domaine résidentiel. L'urbanisme sécuritaire se développe dans des contextes urbains où la criminalité est contrôlée par des interventions dans l'aménagement de la ville. Celles-ci a pour but de décourager les criminels et d'encourager les habitants à surveiller leur environnement. Ce modèle concerne en premier lieu les quartiers résidentiels de la ville, c'est-à-dire la fonction « habiter ». Autour de 1970, alors que la criminalité croissait rapidement dans les zones urbaines aux États-Unis, deux propositions ont commencé à circuler, suggérant une relation entre l'environnement construit et la criminalité.

Oscar Newman (1972) a publié un essai sur l'espace que l'on peut défendre en se concentrant sur les quartiers résidentiels. Cet architecte propose aux habitants des plans pour les espaces publics et privés qui devaient leur donner un sentiment de sécurité pour leurs espaces privés et leur permettre aussi d'assumer leurs responsabilités, tant pour ces espaces privés que pour certaines parties des espaces publics alentour. Il préconisait de subdiviser ces espaces publics de telle façon que chaque partie puisse être placée sous la responsabilité d'un groupe d'habitants 
restreint. Il serait ainsi possible de développer un sens de responsabilité chez tous les habitants pour l'espace public dans son ensemble. L'approche de Newman fut largement acceptée et il conseilla de nombreux gouvernements locaux, aux États-Unis et ailleurs pendant un temps.

Le sous-titre du livre de Newman était Crime Prevention Through Environmental Design. Un livre avait été publié l'année précédente par C.R. Jeffery, professeur de criminologie dans une Université sous le même titre. Cet ouvrage académique proposait un modèle qui mettait en évidence les conditions qui incitaient quelqu'un à commettre un acte criminel, en soulignant l'importance du contexte matériel où il se trouvait. Le livre n'avait pas attiré beaucoup d'attention, mais Newman l'avait remarqué et - en indiquant la source -avait utilisé ce titre comme description additionnelle du contenu de son propre travail.

Plus tard, une association de différents professionnels s'est formée et a pris comme acronyme le nom inventé par Jeffery et popularisé par Newman : «International CPTED Association ». Aux États-Unis, au Royaume-Uni et dans les pays de l'ancien Commonwealth essentiellement, ainsi que dans quelques pays de l'Europe de l'Ouest, le CPTED est un modèle urbain qui peut être utilisé sur demande (http:// www.cpted.net/).

\section{Le développement de la ville comme organisme social}

L'épicentre de l'approche n'est pas la ville comme environnement physique construit, mais la ville comme organisme social pour le dernier modèle présenté dans la typologie (illustration 1). Il s'agit d'un effort pour améliorer la vie urbaine par une mobilisation de l'imagination, une meilleure collaboration et l'incorporation des objets dans les efforts humains, grâce à la connectivité rendue possible par Internet. Une version technologique de ce modèle met l'accent sur la connectivité des gens et des objets rendue possible par l'informatique. Une version imaginative se focalise sur les possibilités d'élargir le potentiel créatif tant au niveau individuel que collectif que la ville permet grâce à la concentration des populations et aux nombreuses possibilités d'interactions qu'elle autorise.

Le modèle de la « ville intelligente " propose une ville où l'informatisation est présente au quotidien dans les quartiers résidentiels et dans les habitations individuelles et où des connections interpersonnelles sont mises en place. Des masses de données sont recueillies sur des plateformes en ligne grâce aux capteurs posés partout et aux téléphones mobiles dont disposent tous les habitants. Cela permet une multitude d'applications qui sont expérimentées avant de les inscrire dans les pratiques des individus et des institutions. Il s'agit d'utilisations plus efficaces de l'infrastructure urbaine (énergie, transport); de nouvelles possibilités d'exprimer simultanément des préférences afin d'améliorer les décisions collectives; et de nouvelles capacités de surveillance et de gestion à distance pour les autorités, les entreprises et les individus.

41 Ce modèle urbain est en pleine expansion dans plusieurs parties du monde. Par exemple, le plan national en Inde prévoit maintenant la construction d'une centaine de villes intelligentes. Ce modèle revêt plusieurs formes. Quelques-unes se concentrent sur un domaine en particulier (par exemple la sécurité), d'autres se développent dans plusieurs domaines simultanément. Il y a des projets dominés par des entreprises télécom et d'autres gérées par des administrations locales, avec l'appui enthousiaste ou 
non de la société civile. Les télécoms opèrent partout dans le monde. Amsterdam Smart City (http://amsterdamsmartcity.com/) est une initiative locale. C'est un projet initié par les autorités locales en 2009 en partenariat avec des dizaines d'institutions, que ce soit des entreprises télécoms, des universités ou des associations. Il y a maintenant 79 projets différents en cours, dont plusieurs font l'effort d'intégrer des groupes sociaux habituellement peu impliqués dans ce type d'activités (personnes âgées, avec un faible niveau scolaire, etc.). Cela pose certains problèmes et, pour le moment, les conclusions ne sont pas encore disponibles. Les questions de fiabilité des données et les risques d'intrusion dans la vie privée font l'objet d'une attention particulière.

L'origine de la ville intelligente se situe dans les études et projets pilotes proposés par William Mitchell et des étudiants du département d'Urban Planning du MIT autour de 2004. Plus tard, ce groupe s'est établi comme le SENSEable City Lab. En Europe, l'expansion en a été facilitée par le financement des Living Labs dans le cadre des programmes de la Commission Européenne (voir à ce sujet, McPhee Chr., Westerlund M. et Leminen S. (eds.), 2012). Mais il y a d'autres applications partout dans le monde. De Nairobi, on retiendra par exemple le cas de l'amélioration de l'efficacité des réseaux de transports publics par l'utilisation des données des téléphones mobiles.

La notion de "ville créative" incarne l'autre volet de cette approche. Après une période de forte suburbanisation et de nombreuses percées dans les centres-villes traditionnels pour les ouvrir à la circulation automobile et pour restructurer des quartiers considérés comme non adaptés aux normes actuelles, une prise de conscience qui repose sur les notions de crise et de désastre urbain apparaît dans les sociétés occidentales autour des années 1970. Une réinterprétation de l'expérience urbaine conduit à une appréciation contraire de la ville, qui s'est ensuite traduite dans un modèle urbain de la ville créative. Initialement abstraction à laquelle on aspirait, la ville créative s'est concrétisée peu à peu autour d'idées conductrices. Un mouvement a émergé avec des dissensions internes mais aussi des contestations qui ont trouvé une solution. Ce sera le CIAM du XXIe siècle.

Durant cette période qui voyait le phénomène urbain remis en cause dans le cercle encore restreint des urbanistes, l'Unesco et le Conseil de l'Europe ont pris des initiatives pour approfondir ces notions par des concours, des prix, des règles de protection, des subventions de projets. Il est aussi à remarquer que, pendant ces mêmes années, l'appréciation des villes par UN-Habitat, organisation déjà évoquée, a changé. Présentées comme des problèmes de premier ordre, elles bénéficient dorénavant d'une image qui les présente comme des chances pour le développement même si des aspects problématiques persistent.

En 1978 au Royaume-Uni, l'urbaniste Charles Landry crée une entreprise de communication pour conseiller les villes en particulier sur leurs identités. Elle s'appelait Commedia, c'est-à-dire dans ce cas Communication et Média. La notion du potentiel créatif d'une communauté locale, et les moyens de le mobiliser et de l'utiliser pour le progrès local furent la ligne directrice de ses conseils dans différents projets. Peter Hall, le célèbre urbaniste académique et fin connaisseur des villes, mais aussi conseiller politique, fut un des collaborateurs occasionnels de Landry, et a beaucoup encouragé ses efforts dans cette direction. En 1990, Landry publiait son inventaire des possibilités pour une mobilisation créative dans une situation particulière avec quelques études de cas (Landry, 1990). 

inouïe des administrations des villes et de leurs communautés locales vers l'extérieur. Les villes n'étaient certes absolument pas isolées auparavant - c'est contre leur nature -, mais l'ouverture actuelle sur le reste du monde implique une densité de communication encore accrue de tous les côtés. L'émergence d'un nouveau modèle urbain est aussitôt connue dans une multitude d'autres villes. Probablement - mais c'est plus difficile à établir rigoureusement -, il y a aussi eu une croissance du nombre des modèles urbains, liée à la croissance du phénomène urbain et à l'augmentation du nombre de professionnels qui travaillent dans ce domaine de développement urbain. 
51 La diffusion de modèles urbains d'une ville à d'autres permet des comparaisons sur les façons de faire ailleurs. Une classification des cas selon la qualité de réalisation du modèle est nécessaire et permet d'identifier des conditions qui facilitent le succès ; une compétition s'ensuit aisément. De telles compétitions sont de plus en plus populaires et soulignent l'intérêt du modèle urbain en créant en quelque sorte une communauté de villes qui sont toutes en train de réaliser le même idéal. Les spécialistes du modèle en question dispensent leurs conseils ou prennent des initiatives en direction des communautés locales et de leurs représentants. Il y a collaboration dans un contexte de compétition. Tous veulent être le cas exemplaire, et faire de leur toponyme la vitrine du modèle.

Dans le même temps, dans chaque ville, les prescriptions du modèle cohabitent avec celles d'autres modèles approuvés dans un contexte local spécifique, toujours différent. Leur inscription dans les pratiques sociales et l'environnement physique urbain modifient le modèle au cours de sa mise en place. Cela permet à chaque ville de s'imaginer unique dans une position-niche. La collaboration est alors possible avec d'autres villes pour échanger les biens et les services rares dont on dispose. Il y a aussi des professionnels, qui peuvent donner des conseils, en sélectionnant dans leur boîte à outils des éléments qui semblent les plus pertinents pour la situation locale. Le processus est identique pour des instances internationales, comme Habitat.

53 Le rêve du modèle Le Corbusier-CIAM a rapidement éclaté. Un seul modèle mondialement disponible et partout utilisé, d'une portée universelle qui comprendrait toutes les activités humaines dans la ville à aménager par des opérations rigoureuses à grande échelle dans l'environnement physique dans un Plan, outil ultra-technologique, a été remplacé par différents modèles urbains qui se sont à leur tour mélangés. On note quand même, dans le bouquet varié qui a vu le jour, des réminiscences occasionnelles, mais jamais de copies intégrales de la ville fonctionnelle.

Le modèle qui promet des espaces sûrs est une élaboration de l'aménagement de l'environnement physique pour un type d'activité (la surveillance pour prévenir des actes criminels) que Le Corbusier avait négligé. Une autre approche via l'environnement physique s'intéresse à la notion de ville durable. La version proposée dans la Charte d'Aalborg prescrit des formes d'urbanisme et des modes de régulation assez détaillés comme le faisait Le Corbusier, mais ces précriptions sont d'inspiration contraire à celles de la Charte d'Athènes. Le caractère technocratique affirmé de la Charte originale est aussi visible dans quelques-uns des modèles qui lui ont succédé. Dans la Gestion Publique Nouvelle (NPM), les connaissances techniques de la nouvelle classe de managers constitueraient un fondement suffisant pour leur confier l'essentiel de la régulation des services publics. Dans le modèle de la ville intelligente, la surveillance permise par la collecte de mégadonnées (ou "big data ») sur des plateformes contrôlées par des spécialistes de plus en plus dépendants de conclusions automatiques, a toutes les chances de conduire à une ville où l'intelligence humaine sera largement remplacée par une technocratie automatique. Le Corbusier avait envisagé la maison comme une machine-à-habiter. La ville dans cette version dystopique de la ville intelligente conduit à une vie urbaine machinisée. Pour l'instant, le mélange de modèles urbains à adapter au contexte local paraît préférable. 


\section{BIBLIOGRAPHY}

Acuto M., 2013. World politics by other means? London, City Diplomacy and the Olympics. The Hague Journal of Diplomacy, 8, p. 287-311.

Boubacha E., 1997. Ville et port. Mutation et recomposition. Note de synthèse et bibliographie. Centre de Documentation de l'Urbanisme, Association Internationale Villes \& Ports, Les éditions Villes \& Territoires, Paris - La Défense.

Corbusier Le, 1942/rééd. 1957, 1971. La Charte d'Athènes. Avec un discours liminaire de Jean Giraudoux suivi d'entretiens avec les étudiants des écoles d'Architecture. Editions de Minuit, Paris.

Etzkowitz H., 2014. The contribution of university-industry-government interactions to creative entrepreneurship and economic development. In R. Sternberg \& G. Krauss (eds.), Handbook of Research on Entrepreneurship and Creativity. E. Edgar Cheltenham, p. 323-359.

Eymeri-Douzans J.-M., Pierre J. (eds.), 2011. Administrative reforms and democratic governance. Routledge London.

Florida R., 2002. The rise of the creative class. Basic Books, New York.

Frey K., Duarte F., 2006. Démocratie participative et gouvernance interactive en Brésil : Santos, Porto Alegre et Curitiba. Espaces et Sociétés, 123, p. 99-112.

Frey K., 2008. Development, good governance and local democracy. Brazilian Political Science Review, 2, 2, p. 39-73.

Friedmann J., 1986. The World City hypothesis. Development and Change, 17, 1, p. 69-83.

Glaeser E.L., 1992. Growth in cities. Journal of Political Economy, 100, 6, p. 1126-1152.

Harding A., Blokland T., 2014. Urban theory. A critical introduction to power, cities and urbanism in the 21st century. Sage London.

Howkins J., 2001. The creative economy. How people make money from ideas. London, Penguin.

Huynh-Quan-Suu C. ,s.a. Etymologie du terme 'gouvernance'. Service de traduction UE.

ICLEI (International Council for Local Environmental Initiatives), 1996. Local Agenda 21 Planning Guide. An introduction to sustainable development planning. ICLEI/IDRC/UNEP, Toronto/Ottowa/ Nairobi.

Independent Evaluation Group World Bank, 2011. An evaluation of the 2007 Strategy and Implementation Plan. World Bank Country-level Engagement on Governance and Anticorruption. Washington 2011, 1-11 (Historical Summary of World Bank Engagement)

Klijn E.-H., 2012. New Public Management and Governance: a comparison. In D. Levi-Faur (ed.), Oxford Handbook of Governance. Oxford University Press Oxford, p. 201-214.

Landry Ch., 2000. Creative city: a toolkit for urban innovators. Earthscan London \& Sterling VA.

Langelier S., 2011. Que reste-t-il de l'expérience pionnière de Porto Alegre. Le Monde Diplomatique, octobre, p. 2-3.

McCann E., Ward K (eds.), 2011. Mobile urbanism. Cities and policymaking in the global age. University of Minnesota Press Minneapolis.

McPhee Chr., Westerlund M., Leminen S. (eds.), 2012. Living Labs special issue, Technology Innovation Management Review, September, p. 6-56. www.timreview.ca 
Markusen A., 2006. Urban development and the politics of a creative class: evidence from a study of artists. Environment \& Planning A 38 october, p. 1921-1940.

Moraga Spà M. de, Botella M. (eds.), 1995. The keys of success: the social, sporting, economic and communications impact of Barcelona '92. Bellaterra Barcelona. http://ceo.uab.cat/2010/docs/ C29501_2.pdf

Musterd S., Murie A. (eds.), 2010. Making competitive cities. Wiley-Blackwell Chichester.

Newman O., 1972. Defensible Space. Crime prevention through urban design. MacMillan, New York.

Peck J., 2010. Mobilizing policy: models, methods and mutations. Geoforum, 41, p. 169-174.

Rossem V. van, 1993. Het Algemeen Uitbreidingsplan van Amsterdam. Geschiedenis en ontwerp. NAi Uitgevers, Rotterdam.

Sassen S., 1991. The global city: New York, London, Tokyo. Princeton University Press, Princeton

Taylor P.J., 2004. World city network: a global urban analysis. Routledge, London \& New York.

Torfing J., 2012. Governance networks. In D. Levi-Faur (ed.), Oxford Handbook of Governance. Oxford University Press Oxford, p. 99-112.

UN-Habitat, 2001. Inclusive Cities Initiative: The Way Forward. Nairobi, UN Habitat.

World Bank, 1991. Managing Development: the Governance dimension. A discussion paper. 34899.

Washington.

http://www.cpted.net/

http://unhabitat.org/about-us/goals-and-strategies-of-un-habitat/

http://amsterdamsmartcity.com/

\section{ABSTRACTS}

Dans le discours public et les pratiques de la gestion urbaine, plusieurs modèles apportent des réponses à la question de savoir comment faire évoluer la/notre ville. Cet article présente une typologie d'une douzaine de ces modèles selon leur portée et leur approche du phénomène urbain. L'origine et la diffusion des modèles sont décrits depuis la ville fonctionnelle de Le Corbusier/CIAM.

\section{INDEX}

Mots-clés: modèle urbain, ville fonctionnelle, urbanisme sécuritaire, ville intelligente, ville créative

Subjects: Sur le Champ - Sur le Terrain

\section{AUTHOR}

\section{HERMAN VAN DER WUSTEN}

Herman van der Wusten, Wusten, H.H.vanderWusten@uva.nl, est Professeur à l'Université d'Amsterdam. 